\title{
Fixed point theorems for cyclic Meir-Keeler type mappings in complete metric spaces
}

\author{
Chi-Ming Chen
}

Correspondence: ming@mail.nhcue. edu.tw

Department of Applied

Mathematics, National Hsinchu University of Education, No.521

Nanda Rd., Hsinchu City 300,

Taiwan

\begin{abstract}
In this article, by using the Meir-Keeler type mappings, we obtain some new fixed point theorems for the cyclic orbital stronger (weaker) Meir-Keeler contractions and generalized cyclic stronger (weaker) Meir-Keeler contractions. Our results generalize or improve many recent fixed point theorems in the literature.

Mathematical Subject Classification: 54H25; 47H10
\end{abstract}

Keywords: generalized cyclic mapping, cyclic orbital mapping, fixed point theorem, cyclic Meir-Keeler contraction

\section{Introduction and preliminaries}

Throughout this article, by $\mathbb{R}^{+}$, we denote the set of all non-negative numbers, while $\mathbb{N}$ is the set of all natural numbers. It is well known and easy to prove that if $(X, d)$ is a complete metric space, and if $f: X \rightarrow X$ is continuous and $f$ satisfies

$$
d\left(f x, f^{2} x\right) \leq k \cdot d(x, f x), \quad \text { for all } x \in X \text { and } k \in(0,1),
$$

then $f$ has a fixed point in $X$. Using the above conclusion, Kirk et al. [1] proved the following fixed point theorem.

Theorem 1 [1] Let $A$ and $B$ be two nonempty closed subsets of a complete metric space $(X, d)$, and suppose $f: A \cup B \rightarrow A \cup B$ satisfies

(i) $f(A) \subset B$ and $f(B) \subset A$,

(ii) $d(f x, f y) \leq k \cdot d(x, y)$ for all $x \in A, y \in B$ and $k \in(0,1)$.

Then $A \cap B$ is nonempty and $f$ has a unique fixed point in $A \cap B$.

The following definitions and results will be needed in the sequel. Let $A$ and $B$ be two nonempty subsets of a metric space $(X, d)$. A mapping $f: A \cup B \rightarrow A \cup B$ is called a cyclic map if $f(A) \subseteq B$ and $f(B) \subseteq A$. In the recent, Karpagam and Agrawal [2] introduced the notion of cyclic orbital contraction, and obtained a unique fixed point theorem for such a map.

Definition 1 [2] Let $A$ and $B$ be nonempty subsets of a metric space $(X, d), f: A \cup B$ $\rightarrow A \cup B$ be a cyclic map such that for some $x \in A$, there exists a $\kappa_{x} \in(0,1)$ such that

$$
d\left(f^{2 n} x, f y\right) \leq k_{x} \cdot d\left(f^{2 n-1} x, y\right), \quad n \in \mathbb{N}, \quad y \in A .
$$

Then $f$ is called a cyclic orbital contraction.

\section{Springer}

(C) 2012 Chen; licensee Springer. This is an Open Access article distributed under the terms of the Creative Commons Attribution License (http://creativecommons.org/licenses/by/2.0), which permits unrestricted use, distribution, and reproduction in any medium, provided the original work is properly cited. 
Theorem 2 [2] Let $A$ and $B$ be two nonempty closed subsets of a complete metric space $(X, d)$, and let $f: A \cup B \rightarrow A \cup B$ be a cyclic orbital contraction. Then $f$ has a fixed point in $A \cap B$.

Furthermore, Kirk et al. [1] introduced the notion of the generalized cyclic mapping and obtained some fixed point results. Let $\left\{A_{i}\right\}_{i=1}^{k}$ be nonempty subsets of a metric space $(X, d)$, and let $f: \cup_{i=1}^{k} A_{i} \rightarrow \cup_{i=1}^{k} A_{i}$ Then $f$ is called a generalized cyclic map if $f$ $\left(A_{i}\right) \subseteq A_{i+1}$ for $i=1,2, \ldots, k$ and $A_{k+1}=A_{1}$. Kirk et al. [1] first extended the question of wherther Edelstein's [3] classical result for contractive mappings, and they obtained the following theorem.

Theorem $3[1]$ Let $\left\{A_{i}\right\}_{i=1}^{k}$ be nonempty closed subsets of a complete metric space $(X, d)$, at least one of which is compact, and suppose $f: \cup_{i=1}^{k} A_{i} \rightarrow \cup_{i=1}^{k} A_{i}$ satisfies the following conditions (where $A_{k+1}=A_{1}$ ):

(i) $f\left(A_{i}\right) \subseteq A_{i+1}$ for $i=1,2, \ldots, k$,

(ii) $d(f x, f y)<d(x, y)$ whenever $x \in A_{\dot{v}} y \in A_{i+1}$ and $x \neq y,(i=1,2, \ldots, k)$.

Then $f$ has a unique fixed point.

On the other hand, Kirk et al. [1] took up the question of whether condition (ii) of Theorem 3 can be replaced by contractive conditions which typically arise in extensions of Banachs theorem. The authors began with a condition introduced by Geraghty [4]. Let $S$ denote the class of those functions $\alpha: \mathbb{R}^{+} \rightarrow[0,1)$ that satisfy the simple condition:

$$
\alpha\left(t_{n}\right) \rightarrow 1 \quad \Rightarrow \quad t_{n} \rightarrow 0 .
$$

Theorem $4[4]$ Let $(X, d)$ be a complete metric space, let $f: X \rightarrow X$, and suppose that there exists $\alpha L S$ such that

$$
d(f x, f y) \leq \alpha(d(x, y)) \cdot d(x, y), \quad \text { for all } x, y \in X .
$$

Then $f$ has a unique fixed point $z$ in $X$ and $\left\{f^{n} x\right\}$ converges to $z$ for each $x \in X$.

Applying Theorem 4, Kirk et al. [1] proved the below theorem.

Theorem 5 [1]Let $\left\{A_{i}\right\}_{i=1}^{k}$ be nonempty closed subsets of a complete metric space $(X, d)$, let $\alpha \in \mathcal{S}$, and suppose $f: \cup_{i=1}^{k} A_{i} \rightarrow \cup_{i=1}^{k} A_{i}$ satisfies the following conditions (where $A_{k+1}$ $\left.=A_{1}\right)$ :

(i) $f\left(A_{i}\right) \subseteq A_{i+1}$ for $i=1,2, \ldots, k$,

(ii) $d(f x, f y) \leq \alpha(d(x, y)) \cdot d(x, y)$ for all $x \in A_{i} y \in A_{i+1}, i=1,2, \ldots, k$.

Then $f$ has a unique fixed point.

In 1969, Boyd and Wong [5] introduced the notion of $\Phi$-contraction. A mapping $f$ : $X \rightarrow X$ on a metric space is called $\Phi$-contraction if there exists an upper semi-continuous function $\psi:[0, \infty) \rightarrow[0, \infty)$ such that

$$
d(f x, f y) \leq \Phi(d(x, y)) \quad \text { for all } x, y \in X .
$$

Kirk et al. [1] also proved the below theorem. 
Theorem $6[1]$ Let $\left\{A_{i}\right\}_{i=1}^{k}$ be nonempty closed subsets of a complete metric space $(X, d)$. Suppose $f: \cup_{i=1}^{k} A_{i} \rightarrow \cup_{i=1}^{k} A_{i}$ satisfies the following conditions (where $A_{k+1}=A_{1}$ ):

(i) $f\left(A_{i}\right) \subseteq A_{i+1}$ for $i=1,2, \ldots, k$,

(ii) $d(f x, f y) \leq \Phi(d(x, y))$ for all $x \in A_{i} y \in A_{i+1}, i=1,2, \ldots, k$,

where $\Phi:[0, \infty) \rightarrow[0, \infty)$ is upper semi-contionuous from the right and satisfies $0 \leq$ $\psi(t)<t$ for $t>0$. Then $f$ has a unique fixed point.

In this article, we also recall the notion of the Meir-Keeler type mapping. A function $\psi: \mathbb{R}^{+} \rightarrow \mathbb{R}^{+}$is said to be a Meir-Keeler type mapping (see [6]), if for each $\eta \in \mathbb{R}^{+}$, there exists $\delta>0$ such that for $t \in \mathbb{R}^{+}$with $\eta \leq t<\eta+\delta$, we have $\psi(t)<\eta$. Subsequently, some authors worked on this notion (for example, [7-10]). This article will deal with two new mappings of the stronger Meir-Keeler type and weaker Meir-Keeler type in a metric space $(X, d)$. We first introduce the below notion of stronger MeirKeeler type mapping in a metric space.

Definition 2 Let $(X, d)$ be a metric space. We call $\psi: \mathbb{R}^{+} \rightarrow[0,1)$ a stronger MeirKeeler type mapping in $X$ if the mapping $\psi$ satisfies the following condition:

$$
\forall \eta>0 \quad \exists \delta>0 \quad \exists \gamma_{\eta} \in[0,1) \quad \forall x, y \in X\left(\eta \leq d(x, y)<\delta+\eta \quad \Rightarrow \quad \psi(d(x, y))<\gamma_{\eta}\right) .
$$

Example 1 Let $X=\mathbb{R}^{2}$ and we define $d: X \times X \rightarrow \mathbb{R}^{+}$by

$$
d(x, y)=\left|x_{1}-y_{1}\right|+\left|x_{2}-y_{2}\right| \quad \text { for all } x=\left(x_{1}, x_{2}\right), \quad y=\left(y_{1}, y_{2}\right) \in X
$$

If $\psi: \mathbb{R}^{+} \rightarrow[0,1), \psi(d(x, y))=\frac{d(x, y)}{d(x, y)+1}$, then $\psi$ is a stronger Meir-Keeler type mapping in $X$.

The following provides an example of a Meir-Keeler type mapping which is not a stronger Meir-Keeler type mapping in a metric space $(X, d)$.

Example 2 Let $X=\mathbb{R}^{2}$ and we define $d: X \times X \rightarrow \mathbb{R}^{+}$by

$$
\begin{gathered}
d(x, y)=\left|x_{1}-y_{1}\right|+\left|x_{2}-y_{2}\right| \quad \text { for all } x=\left(x_{1}, x_{2}\right), \quad y=\left(y_{1}, y_{2}\right) \in X . \\
\text { If } \phi: \mathbb{R}^{+} \rightarrow \mathbb{R}^{+}, \\
\varphi(d(x, y))= \begin{cases}d(x, y)-1, & \text { if } d(x, y)>1 ; \\
0, & \text { if } d(x, y) \leq 1,\end{cases}
\end{gathered}
$$

then $\phi$ is a Meir-Keeler type mapping which is not a stronger Meir-Keeler type mapping in $X$.

We next introduce the below notion of weaker Meir-Keeler type mapping in a metric space.

Definition 3 Let $(X, d)$ be a metric space, and $\phi: \mathbb{R}^{+} \rightarrow \mathbb{R}^{+}$. Then $\phi$ is called a weaker Meir-Keeler type mapping in $X$, if the mapping $\phi$ satisfies the following condition:

$$
\forall \eta>0 \exists \delta>0 \quad \forall x, y \in X \quad\left(\eta \leq d(x, y)<\delta+\eta \Rightarrow \exists n_{0} \in \mathbb{N} \varphi^{n_{0}}(d(x, y))<\eta\right) .
$$

Example 3 Let $X=\mathbb{R}^{2}$ and we define $d: X \times X \rightarrow \mathbb{R}^{+}$by

$$
d(x, y)=\left|x_{1}-y_{1}\right|+\left|x_{2}-y_{2}\right| \quad \text { for all } x=\left(x_{1}, x_{2}\right), \quad y=\left(y_{1}, y_{2}\right) \in X .
$$


If $\phi: \mathbb{R}^{+} \rightarrow \mathbb{R}^{+}, \varphi(d(x, y))=\frac{1}{2} d(x, y)$, then $\phi$ is a weaker Meir-Keeler type mapping in $X$.

The following provides an example of a weaker Meir-Keeler type mapping which is not a Meir-Keeler type mapping in a metric space $(X, d)$.

Example 4 Let $X=\mathbb{R}^{2}$ and we define $d: X \times X \rightarrow \mathbb{R}^{+}$by

$$
d(x, y)=\left|x_{1}-y_{1}\right|+\left|x_{2}-y_{2}\right| \quad \text { for all } x=\left(x_{1}, x_{2}\right), \quad y=\left(y_{1}, y_{2}\right) \in X
$$

If $\phi: \mathbb{R}^{+} \rightarrow \mathbb{R}^{+}$,

$$
\varphi(d(x, y))= \begin{cases}0, & \text { if } d(x, y) \leq 1 \\ 2 \cdot d(x, y), & \text { if } 1<d(x, y)<2 \\ 1, & \text { if } d(x, y) \geq 2\end{cases}
$$

then $\phi$ is a weaker Meir-Keeler type mapping which is not a Meir-Keeler type mapping in $X$.

\section{The fixed point theorems for cyclic orbital Meir-Keeler contractions}

Using the notions of the cyclic orbital contraction (see, Definition 1) and stronger Meir-Keeler type mapping (see, Definition 2), we introduce the below notion of cyclic orbital stronger Meir-Keeler contraction.

Definition 4 Let $A$ and $B$ be nonempty subsets of a metric space $(X, d)$. Suppose $f: A$ $\cup B \rightarrow A \cup B$ is a cyclic map such that for some $x \in A$, there exists a stronger MeirKeeler type mapping $\psi: \mathbb{R}^{+} \rightarrow[0,1)$ in $X$ such that

$$
d\left(f^{2 n} x, f y\right) \leq \psi\left(d\left(f^{2 n-1} x, y\right)\right) \cdot d\left(f^{2 n-1} x, y\right), \quad n \in \mathbb{N}, \quad y \in A .
$$

Then $f$ is called a cyclic orbital stronger Meir-Keeler $\psi$-contraction.

Now, we are in a position to state the following theorem.

Theorem 7 Let $A$ and $B$ be two nonempty closed subsets of a complete metric space $(X, d)$, and let $\psi: \mathbb{R}^{+} \rightarrow[0,1)$ be a stronger Meir-Keeler type mapping in $X$. Suppose $f:$ $A \cup B \rightarrow A \cup B$ is a cyclic orbital stronger Meir-Keeler $\psi$-contraction. Then $A \cap B$ is nonempty and $f$ has a unique fixed point in $A \cap B$.

Proof. Since $f: A \cup B \rightarrow A \cup B$ is a cyclic orbital stronger Meir-Keeler $\psi$-contraction, there exists $x \in A$ satisfying (2), and we also have that for each $n \in \mathbb{N}$,

$$
\begin{aligned}
d\left(f^{2 n} x, f^{2 n+1} x\right) & \left.\leq \psi d\left(f^{2 n-1} x, f^{2 n} x\right)\right) \cdot d\left(f^{2 n-1} x, f^{2 n} x\right) \\
& \leq d\left(f^{2 n-1} x, f^{2 n} x\right)
\end{aligned}
$$

and

$$
\begin{aligned}
d\left(f^{2 n+1} x, f^{2 n+2} x\right) & =d\left(f^{2 n+2} x, f^{2 n+1} x\right) \\
& \leq \psi\left(d\left(f^{2 n+1} x, f^{2 n} x\right)\right) \cdot d\left(f^{2 n+1} x, f^{2 n} x\right) \\
& \leq d\left(f^{2 n+1} x, f^{2 n} x\right)=d\left(f^{2 n} x, f^{2 n+1} x\right) .
\end{aligned}
$$

Generally, we have

$$
d\left(f^{n} x, f^{n+1} x\right) \leq d\left(f^{n-1} x, f^{n} x\right), \quad n \in \mathbb{N} .
$$

Thus the sequence $\left\{d\left(f^{n} x, f^{n+1} x\right)\right\}$ is non-increasing and hence it is convergent. Let $\lim _{n \rightarrow \infty} d\left(f^{n} x, f^{n+1} x\right)=\eta$. Then there exists $\kappa_{0} \in \mathbb{N}$ and $\delta>0$ such that for all $n \geq \kappa_{0}$, 


$$
\eta \leq d\left(f^{n} x, f^{n+1} x\right)<\eta+\delta .
$$

Taking into account the above inequality and the definition of stronger Meir-Keeler type mapping $\psi$ in $X$, corresponding to $\eta$ use, there exists $\gamma_{\eta} \in[0,1)$ such that

$$
\psi\left(d\left(f^{k_{0}+n} x, f^{k_{0}+n+1} x\right)\right)<\gamma_{\eta} \text { for all } n \in \mathbb{N} \cup\{0\} .
$$

Therefore, by (2), we also deduce that for each $n \in \mathbb{N}$,

$$
\begin{aligned}
d\left(f^{k_{0}+n} x, f^{k_{0}+n+1} x\right) & \leq \psi\left(d\left(f^{k_{0}+n-1} x, f^{k_{0}+n} x\right)\right) \cdot d\left(f^{k_{0}+n-1} x, f^{k_{0}+n} x\right) \\
& <\gamma_{\eta} \cdot d\left(f^{k_{0}+n-1} x, f^{k_{0}+n} x\right),
\end{aligned}
$$

and it follows that for each $n \in \mathbb{N}$,

$$
\begin{aligned}
d\left(f^{k_{0}+n} x, f^{k_{0}+n+1} x\right) & <\gamma_{\eta} \cdot d\left(f^{k_{0}+n-1} x, f^{k_{0}+n} x\right) \\
& <\cdots \cdots \\
& <\gamma_{\eta}^{n} \cdot d\left(f^{k_{0}} x, f^{k_{0}+1} x\right) .
\end{aligned}
$$

So

$$
\lim _{n \rightarrow \infty} d\left(f^{k_{0}+n} x, f^{k_{0}+n+1} x\right)=0, \quad \text { since } \quad \gamma_{\eta} \in[0,1) .
$$

We now claim that $\lim _{n \rightarrow \infty} d\left(f^{k_{0}+n} x, f^{k_{0}+m} x\right)=0$ for $m>n$. For $m, n \in \mathbb{N}$ with $m>$ $n$, we have

$$
d\left(f^{k_{0}+n} x, f^{k_{0}+m} x\right) \leq \sum_{i=n}^{m-1} d\left(f^{k_{0}+i} x, f^{k_{0}+i+1} x\right)<\frac{\gamma_{\eta}^{m-1}}{1-\gamma_{\eta}} d\left(f^{k_{0}} x, f^{k_{0}+1} x\right),
$$

and hence $d\left(f^{n} x, f^{n} x\right) \rightarrow 0$, since $0<\gamma_{\eta}<1$. So $\left\{f^{n} x\right\}$ is a Cauchy sequence. Since (X, $d$ ) is a complete metric space, there exists $v \in A \cup B$ such that $\lim _{n \rightarrow \infty} f^{n} x=v$. Now $\left\{f^{2 n} x\right\}$ is a sequence in $A$ and $\left\{f^{2 n-1} x\right\}$ is a sequence in $B$, and also both converge to $v$. Since $A$ and $B$ are closed, $v \in A \cap B$, and so $A \cap B$ is nonempty. Since

$$
\begin{aligned}
d(v, f v) & =\lim _{n \rightarrow \infty} d\left(f^{2 n} x, f v\right) \\
& \leq \lim _{n \rightarrow \infty}\left[\psi\left(d\left(f^{2 n-1} x, v\right)\right) \cdot d\left(f^{2 n-1} x, v\right)\right] \\
& \leq \lim _{n \rightarrow \infty}\left[\gamma_{\eta} \cdot d\left(f^{2 n-1} x, v\right)\right]=0,
\end{aligned}
$$

hence $v$ is a fixed point of $f$.

Finally, to prove the uniqueness of the fixed point, let $\mu$ be another fixed point of $f$. By the cyclic character of $f$, we have $v, \mu \in A \cap B$. Since $f$ is a cyclic orbital stronger Meir-Keeler $\psi$-contraction, we have

$$
\begin{aligned}
d(\nu, \mu) & =d(v, f \mu)=\lim _{n \rightarrow \infty} d\left(f^{2 n} x, f \mu\right) \\
& \leq \lim _{n \rightarrow \infty}\left[\psi\left(d\left(f^{2 n-1} x, \mu\right)\right) \cdot d\left(f^{2 n-1} x, \mu\right)\right] \\
& \leq \lim _{n \rightarrow \infty}\left[\gamma_{\eta} \cdot d\left(f^{2 n-1} x, \mu\right)\right] \\
& \leq \gamma_{\eta} \cdot d(v, \mu)<d(v, \mu),
\end{aligned}
$$

a contradiction. Therefore $\mu=v$, and so $v$ is a unique fixed point of $f$. 
Example 5 Let $A=B=X=\mathbb{R}^{+}$and we define $d: X \times X \rightarrow \mathbb{R}^{+}$by

$$
d(x, y)=|x-y|, \quad \text { for } x, y \in X
$$

Define $f: X \rightarrow X$ by

$$
f(x)=\left\{\begin{array}{l}
0, \text { if } 0 \leq x<1 \\
\frac{1}{4}, \text { if } x \geq 1
\end{array}\right.
$$

and define $\psi: \mathbb{R}^{+} \rightarrow[0,1)$ by

$$
\psi(t)= \begin{cases}\frac{1}{3}, & \text { if } 0 \leq t \leq 1 ; \\ \frac{t}{t+1}, & \text { if } t>1\end{cases}
$$

Then $f$ is a cyclic orbital stronger Meir-Keeler $\psi$-contraction and 0 is the unique fixed point.

Using the notions of the cyclic orbital contraction (see, Definition 1) and weaker Meir-Keeler type mapping (see, Definition 3), we next introduce the notion of cyclic orbital weaker Meir-Keeler contraction. We first define the below notion of $\phi$-mapping.

Definition 5 Let $(X, d)$ be a metric space. We call $\phi: \mathbb{R}^{+} \rightarrow \mathbb{R}^{+}$a $\phi$-mapping in $X$ if the function $\phi$ satisfies the following conditions:

$\left(\phi_{1}\right) \phi>$ is a weaker Meir-Keeler type mapping in $X$ with $\phi(0)=0$;

$\left(\phi_{2}\right)(a)$ if $\lim _{n \rightarrow \infty} t_{n}=\gamma>0$, then $\lim _{n \rightarrow \infty} \phi\left(t_{n}\right)<\gamma$, and

(b) if $\lim _{n \rightarrow \infty} t_{n}=0$, then $\lim _{n \rightarrow \infty} \phi\left(t_{n}\right)=0$;

$\left(\phi_{3}\right)\left\{\phi^{n}(t)\right\}_{n \in \mathbb{N}}$ is decreasing.

Definition 6 Let $A$ and $B$ be nonempty subsets of a metric space $(X, d)$. Suppose $f: A$ $\cup B \rightarrow A \cup B$ is a cyclic map such that for some $x \in A$, there exists a $\phi$-mapping $\phi: \mathbb{R}$ ${ }^{+} \rightarrow \mathbb{R}^{+}$in $X$ such that

$$
d\left(f^{2 n} x, f y\right) \leq \varphi\left(d\left(f^{2 n-1} x, y\right)\right), \quad n \in \mathbb{N}, \quad y \in A
$$

Then $f$ is called a cyclic orbital weaker Meir-Keeler $\phi$-contraction.

Now, we are in a position to state the following theorem.

Theorem 8 Let $A$ and $B$ be two nonempty closed subsets of a complete metric space $(X, d)$, and let $\phi: \mathbb{R}^{+} \rightarrow \mathbb{R}^{+}$be a $\phi$-mapping in $X$. Suppose $f: A \cup B \rightarrow A \cup B$ is a cyclic orbital weaker Meir-Keeler $\phi$-contraction. Then $A \cap B$ is nonempty and $f$ has a unique fixed point in $A \cap B$.

Proof. Since $f: A \cup B \rightarrow A \cup B$ is a cyclic orbital weaker Meir-Keeler $\phi$-contraction, there exists $x \in A$ satisfying (3), and we also have that for each $n \in \mathbb{N}$,

$$
d\left(f^{2 n} x, f^{2 n+1} x\right) \leq \varphi\left(d\left(f^{2 n-1} x, f^{2 n} x\right)\right),
$$

and

$$
\begin{aligned}
d\left(f^{2 n+1} x, f^{2 n+2} x\right) & =d\left(f^{2 n+2} x, f^{2 n+1} x\right) \\
& \leq \varphi\left(d\left(f^{2 n+1} x, f^{2 n} x\right)\right)
\end{aligned}
$$


Generally, we have

$$
d\left(f^{n} x, f^{n+1} x\right) \leq \varphi\left(d\left(f^{n-1} x, f^{n} x\right)\right), \quad n \in \mathbb{N} .
$$

So we conclude that for each $n \in \mathbb{N}$

$$
\begin{aligned}
d\left(f^{n} x, f^{n+1} x\right) & \leq \varphi\left(d\left(f^{n-1} x, f^{n} x\right)\right) \\
& \leq \varphi^{2}\left(d\left(f^{n-2} x, f^{n-1} x\right)\right) \\
& \leq \cdots \cdots \\
& \leq \varphi^{n}(d(x, f x)) .
\end{aligned}
$$

Since $\left\{\phi^{n}(d(x, f x))\right\}_{n \in \mathbb{N}}$ is decreasing, it must converge to some $\eta \geq 0$. We claim that $\eta=0$. On the contrary, assume that $\eta>0$. Then by the definition of weaker Meir-Keeler type mapping $\phi$ in $X$, there exists $\delta>0$ such that for $x, y \in X$ with $\eta \leq d(x, y)<\delta+$ $\eta$, there exists $n_{0} \in \mathbb{N}$ such that $\varphi^{n_{0}}(d(x, y))<\eta$. Since $\lim _{n \rightarrow \infty} \phi^{n}(d(x, f x))=\eta$, there exists $m_{0} \in \mathbb{N}$ such that $\eta \leq \phi^{m}(d(x, f x))<\delta+\eta$, for all $m>m_{0}$. Thus, we conclude that $\varphi^{m_{0}+n_{0}}\left(d\left(x_{0}, x_{1}\right)\right)<\eta$, and we get a contradiction. So $\lim _{n \rightarrow \infty} \phi^{n}(d(x, f x))=0$, that is, $\lim _{n \rightarrow \infty} d\left(\rho^{n} x, f^{n+1} x\right)=0$.

Next, we let $c_{m}=d\left(f^{m} x, f^{m+1} x\right)$, and we claim that the following result holds:

for each $\varepsilon>0$, there is $n_{0}(\varepsilon) \in \mathbb{N}$ such that for all $m, n \geq n_{0}(\varepsilon)$,

$$
d\left(f^{m} x, f^{m+1} x\right)<\varepsilon .
$$

We shall prove $\left(^{*}\right)$ by contradiction. Suppose that $\left(^{*}\right)$ is false. Then there exists some $\varepsilon>0$ such that for all $p \in \mathbb{N}$, there are $m_{p}, n_{p} \in \mathbb{N}$ with $m_{p}>n_{p} \geq p$ satisfying:

(i) $m_{p}$ is even and $n_{p}$ is odd,

(ii) $d\left(f^{m_{p}} x, f^{n_{p}} x\right) \geq \varepsilon$, and

(iii) $m_{p}$ is the smallest even number such that the conditions (i), (ii) hold.

Since $c_{m} \searrow 0$, by $(i i)$, we have $\lim _{k \rightarrow \infty} d\left(f^{m_{p}} x, f^{n_{p}} x\right)=\varepsilon$, and

$$
\begin{aligned}
\varepsilon & \leq d\left(f^{m_{p}} x, f^{n_{p}} x\right) \\
& \leq d\left(f^{m_{p}} x, f^{m_{p}+1} x\right)+d\left(f^{m_{p}+1} x, f^{n_{p}+1} x\right)+d\left(f^{n_{p}+1} x, f^{n_{p}} x\right) \\
& \leq d\left(f^{m_{p}} x, f^{m_{p}+1} x\right)+\varphi\left(d\left(f^{m_{p}} x, f^{n_{p}} x\right)\right)+d\left(f^{n_{p}+1} x, f^{n_{p}} x\right) .
\end{aligned}
$$

Letting $p \rightarrow \infty$. Then by the condition $\left(\phi_{2}\right)-(a)$ of $\phi$-mapping, we have

$$
\varepsilon \leq 0+\lim _{p \rightarrow \infty} \varphi\left(d\left(f^{m_{p}} x, f^{n_{p}} x\right)\right)+0<\varepsilon
$$

a contradiction. So $\left\{f^{n} x\right\}$ is a Cauchy sequence. Since $(X, d)$ is a complete metric space, there exists $v \in A \cup B$ such that $\lim _{n \rightarrow \infty} f_{n} x=v$. Now $\left\{f^{2 n} x\right\}$ is a sequence in $A$ and $\left\{f^{2 n-1} x\right\}$ is a sequence in $B$, and also both converge to $v$. Since $A$ and $B$ are closed, $v \in A \cap B$, and so $A \cap B$ is nonempty. By the condition $\left(\phi_{2}\right)-(b)$ of $\phi$-mapping, we have

$$
\begin{aligned}
d(v, f v) & =\lim _{n \rightarrow \infty} d\left(f^{2 n} x, f v\right) \\
& \leq \lim _{n \rightarrow \infty} \varphi\left(d\left(f^{2 n-1} x, v\right)\right)=0
\end{aligned}
$$

hence $v$ is a fixed point of $f$. Let $\mu$ be another fixed point of $f$. Since $f$ is a cyclic orbital weaker Meir-Keeler $\phi$-contraction, we have 


$$
\begin{aligned}
d(v, \mu) & =d(\nu, f \mu)=\lim _{n \rightarrow \infty} d\left(f^{2 n} x, f \mu\right) \\
& \leq \lim _{n \rightarrow \infty} \varphi\left(d\left(f^{2 n-1} x, \mu\right)\right) \\
& <d(v, \mu),
\end{aligned}
$$

a contradiction. Therefore, $\mu=v$. Thus $v$ is a unique fixed point of $f$.

Example 6 Let $A=B=X=\mathbb{R}^{+}$and we define $d: X \times X \rightarrow \mathbb{R}^{+}$by

$$
d(x, y)=|x-y|, \quad \text { for } x, y \in X .
$$

Define $f: X \rightarrow X$ by

$$
f(x)=\left\{\begin{array}{l}
0, \text { if } 0 \leq x<1 \\
\frac{1}{4}, \text { if } x \geq 1
\end{array}\right.
$$

and define $\phi: \mathbb{R}^{+} \rightarrow \mathbb{R}^{+}$by

$$
\varphi(t)=\frac{1}{3} t \quad \text { for } t \in \mathbb{R}^{+} .
$$

Then $f$ is a cyclic orbital weaker Meir-Keeler $\phi$-contraction and 0 is the unique fixed point.

\section{The fixed point theorems for generalized cyclic Meir-Keeler contractions}

Using the notions of the generalized cyclic contraction [1] and stronger Meir-Keeler type mapping, we introduce the below notion of generalized cyclic stronger Meir-Keeler contraction.

Definition 7 Let $\left\{A_{i}\right\}_{i=1}^{k}$ be nonempty subsets of a metric space $(X, d)$, let $\psi: \mathbb{R}^{+} \rightarrow$ $[0,1)$ be a stronger Meir-Keeler type mapping in $X$, and suppose $f: \cup_{i=1}^{k} A_{i} \rightarrow \cup_{i=1}^{k} A_{i}$ satisfies the following conditions (where $A_{k+1}=A_{1}$ ):

(i) $f\left(A_{i}\right) \subseteq A_{i+1}$ for $i=1,2, \ldots, k$;

(ii) $d(f x, f y) \leq \psi(d(x, y)) \cdot d(x, y)$ for all $x \in A_{i} y \in A_{i+1}, i=1,2, \ldots, k$.

Then we call $f$ a generalized cyclic stronger Meir-Keeler $\psi$-contraction.

We state the main fixed point theorem for the generalized cyclic stronger Meir-Keeler $\psi$-contraction, as follows:

Theorem 9 Let $\left\{A_{i}\right\}_{i=1}^{k}$ be nonempty closed subsets of a complete metric space $(X, d)$, let $\psi: \mathbb{R}^{+} \rightarrow[0,1)$ be a stronger Meir-Keeler type mapping in $X$, and let $f: \cup_{i=1}^{k} A_{i} \rightarrow \cup_{i=1}^{k} A_{i}$ be a generalized cyclic stronger Meir-Keeler $\psi$-contraction. Then $f$ has a unique fixed point in $\cap_{i=1}^{k} A_{i}$.

Proof. Given $x_{0} \in X$ and let $x_{n}=f^{n} x_{0}, n \in \mathbb{N}$. Since $f$ is a generalized cyclic stronger Meir-Keeler $\psi$-contraction, we have that for each $n \in \mathbb{N}$

$$
\begin{aligned}
d\left(x_{n}, x_{n+1}\right) & =d\left(f^{n} x_{0}, f^{n+1} x_{0}\right) \\
& \leq \psi\left(d\left(f^{n-1} x_{0}, f^{n} x_{0}\right)\right) \cdot d\left(f^{n-1} x_{0}, f^{n} x_{0}\right) \\
& \leq d\left(f^{n-1} x_{0}, f^{n} x_{0}\right)=d\left(x_{n-1}, x_{n}\right) .
\end{aligned}
$$

Thus the sequence $\left\{d\left(x_{n}, x_{n+1}\right)\right\}$ is non-increasing and hence it is convergent. Let $\lim _{n \rightarrow \infty} d\left(x_{w}, x_{n+1}\right)=\eta \geq 0$. Then there exists $\kappa_{0} \in \mathbb{N}$ and $\delta>0$ such that for all $n \geq \kappa_{0}$ 


$$
\eta \leq d\left(x_{n}, x_{n+1}\right)<\eta+\delta .
$$

Taking into account the above inequality and the definition of stronger Meir-Keeler type mapping $\psi$ in $X$, corresponding to $\eta$ use, there exists $\gamma_{n} \in[0,1)$ such that

$$
\psi\left(d\left(x_{k_{0}+n}, x_{k_{0}+n+1}\right)\right)<\gamma_{\eta}
$$

for all $n \in \mathbb{N} \cup\{0\}$. Thus, we can deduce that for each $n \in \mathbb{N}$

$$
\begin{aligned}
d\left(x_{k_{0}+n}, x_{k_{0}+n+1}\right) & =d\left(f^{k_{0}+n} x_{0}, f^{k_{0}+n+1} x_{0}\right) \\
& \leq \psi\left(d\left(f^{k_{0}+n-1} x_{0}, f^{k_{0}+n} x_{0}\right)\right) \cdot d\left(f^{k_{0}+n-1} x_{0}, f^{k_{0}+n} x_{0}\right) \\
& <\gamma_{\eta} d\left(f^{k_{0}+n-1} x_{0}, f^{k_{0}+n} x_{0}\right),
\end{aligned}
$$

and it follows that for each $n \in \mathbb{N}$

$$
\begin{aligned}
d\left(x_{k_{0}+n}, x_{k_{0}+n+1}\right) & <\gamma_{\eta} d\left(f^{k_{0}+n-1} x_{0}, f^{k_{0}+n} x_{0}\right) \\
& <\cdots \\
& <\gamma_{\eta}^{n} d\left(f^{k_{0}+1} x_{0}, f^{k_{0}+2} x_{0}\right) .
\end{aligned}
$$

So

$$
\lim _{n \rightarrow \infty} d\left(x_{k_{0}+n}, x_{k_{0}+n+1}\right)=0, \quad \text { since } \quad \gamma_{\eta}<1 .
$$

We now claim that $\lim _{n \rightarrow \infty} d\left(x_{k_{0}+n}, x_{k_{0}+m}\right)=0$ for $m>n$. For $m, n \in \mathbb{N}$ with $m>n$, we have

$$
\begin{aligned}
d\left(x_{k_{0}+n}, x_{k_{0}+m}\right) & =d\left(f^{k_{0}+n} x_{0}, f^{k_{0}+m} x_{0}\right) \\
& \leq \sum_{i=n}^{m-1} d\left(f^{k_{0}+i} x_{0}, f^{k_{0}+i+1} x_{0}\right) \\
& \left.<\frac{\gamma_{\eta}^{m-1}}{1-\gamma_{\eta}} d\left(f^{k_{0}} x_{0}, f^{k_{0}+1} x_{0}\right)\right),
\end{aligned}
$$

and hence $d\left(f^{n} x_{0}, f^{m} x_{0}\right) \rightarrow 0$, since $0<\gamma_{\eta}<1$. So $\left\{f^{n} x_{0}\right\}$ is a Cauchy sequence. Since $X$ is complete, there exists $v \in \cup_{i=1}^{k} A_{i}$ such that $\lim _{n \rightarrow \infty} f^{n} x_{0}=v$. Now for all $i=0,1,2, \ldots$, $k-1,\left\{f^{k n-i} x\right\}$ is a sequence in $A_{i}$ and also all converge to $v$. Since $A_{i}$ is clsoed for all $i=$ $1,2, \ldots, k$, we conclude $v \in \cap_{i=1}^{k} A_{i}$, and also we conclude that $\cap_{i=1}^{k} A_{i} \neq \phi$ Since

$$
\begin{aligned}
d(v, f v) & =\lim _{n \rightarrow \infty} d\left(f^{k n} x, f v\right) \\
& \leq \lim _{n \rightarrow \infty}\left[\psi\left(d\left(f^{k n-1} x, v\right)\right) \cdot d\left(f^{k n-1} x, v\right)\right] \\
& \leq \lim _{n \rightarrow \infty}\left[\gamma_{\eta} \cdot d\left(f^{k n-1} x, v\right)\right]=0
\end{aligned}
$$

hence $v$ is a fixed point of $f$.

Finally, to prove the uniqueness of the fixed point, let $\mu$ be another fixed point of $f$. By the cyclic character of $f$, we have $\mu \in \cap_{i=1}^{k} A_{i}$. Since $f$ is a generalized cyclic stronger Meir-Keeler $\psi$-contraction, we have 


$$
\begin{aligned}
d(\nu, \mu) & =d(\nu, f \mu)=\lim _{n \rightarrow \infty} d\left(f^{k n} x, f \mu\right) \\
& \leq \lim _{n \rightarrow \infty}\left[\psi\left(d\left(f^{k n-1} x, \mu\right)\right) \cdot d\left(f^{k n-1} x, \mu\right)\right] \\
& \leq \lim _{n \rightarrow \infty}\left[\gamma_{\eta} \cdot d\left(f^{k n-1} x, \mu\right)\right] \\
& \leq \gamma_{\eta} \cdot d(v, \mu)<d(\nu, \mu)
\end{aligned}
$$

a contradiction. Therefore, $\mu=v$. Thus $v$ is a unique fixed point of $f$.

Example 7 Let $X=\mathbb{R}^{3}$ and we define $d: X \times X \rightarrow \mathbb{R}^{+}$by

$$
d(x, y)=\left|x_{1}-y_{1}\right|+\left|x_{2}-y_{2}\right|+\left|x_{3}-y_{3}\right|, \quad \text { for } x=\left(x_{1}, x_{2}, x_{3}\right), y=\left(y_{1}, y_{2}, y_{3}\right) \in X,
$$

and let $A=\{(x, 0,0): x \in \mathbb{R}\}, B=\{(0, y, 0): y \in \mathbb{R}\}, C=\{(0,0, z): z \in \mathbb{R}\}$ be three subsets of $X$. Define $f: A \cup B \cup C \rightarrow A \cup B \cup C$ by

$$
\begin{array}{ll}
f((x, 0,0))=(0, x, 0) ; & \text { for all } x \in \mathbb{R} ; \\
f((0, y, 0))=(0,0, y) ; & \text { for all } y \in \mathbb{R} ; \\
f((0,0, z))=(z, 0,0) ; & \text { for all } z \in \mathbb{R} .
\end{array}
$$

and define $\psi: \mathbb{R}^{+} \rightarrow[0,1)$ by

$$
\psi(t)=\frac{t}{t+1} \quad ; \text { for } t \in \mathbb{R}^{+} .
$$

Then $f$ is a generalized cyclic stronger Meir-Keeler $\psi$-contraction and $(0,0,0)$ is the unique fixed point.

Using the notions of the generalized cyclic contraction and weaker Meir-Keeler type mapping, we introduce the below notion of generalized cyclic weaker Meir-Keeler contraction.

Definition 8 Let $\left\{A_{i}\right\}_{i=1}^{k}$ be nonempty subsets of a metric space $(X, d)$, let $\phi: \mathbb{R}^{+} \rightarrow \mathbb{R}^{+}$ be a $\phi$-mapping in $X$, and suppose $f: \cup_{i=1}^{k} A_{i} \rightarrow \cup_{i=1}^{k} A_{i}$ satisfies the following conditions (where $A_{k+1}=A_{1}$ ):

(i) $f\left(A_{i}\right) \subseteq A_{i+1}$ for $i=1,2, \ldots, k$;

(ii) $d(f x, f y) \leq \phi(d(x, y))$ for all $x \in A_{i} y \in A_{i+1}, i=1,2, \ldots, k$.

Then we call $f$ a generalized cyclic weaker Meir-Keeler $\phi$-contraction.

Now, we are in a position to state the following theorem.

Theorem 10 Let $\left\{A_{i}\right\}_{i=1}^{k}$ be nonempty closed subsets of a complete metric space $(X, d)$, let $\phi: \mathbb{R}^{+} \rightarrow \mathbb{R}^{+}$be a $\phi$-mapping in $X$, and let $f:$ cup $p_{i=1}^{k} A_{i} \rightarrow \cup_{i=1}^{k} A_{i}$ be a generalized cyclic weaker Meir-Keeler $\phi$-contraction. Then $f$ has a unique fixed point in $\cap_{i=1}^{k} A_{i}$.

Proof. Given $x_{0} \in X$ and let $x_{n}=f^{n} x_{0}, n \in \mathbb{N}$. Since $f$ is a generalized cyclic weaker Meir-Keeler $\phi$-contraction, we have that for each $n \in \mathbb{N}$

$$
\begin{aligned}
d\left(x_{n}, x_{n+1}\right) & =d\left(f^{n} x_{0}, f^{n+1} x_{0}\right) \\
& \leq \varphi\left(d\left(f^{n-1} x_{0}, f^{n} x_{0}\right)\right)=\varphi\left(d\left(x_{n-1}, x_{n}\right)\right) \\
& \leq \cdots \cdots \\
& \leq \varphi^{n}\left(d\left(x_{0}, x_{1}\right)\right) .
\end{aligned}
$$


Since $\left\{\phi^{n}\left(d\left(x_{0}, x_{1}\right)\right)\right\}_{n \in \mathbb{N}}$ is decreasing, it must converge to some $\eta \geq 0$. We claim that $\eta=0$. On the contrary, assume that $\eta>0$. Then by the definition of weaker Meir-Keeler type mapping $\phi$ in $X$, there exists $\delta>0$ such that for $x, y \in X$ with $\eta \leq d(x, y)<\delta+$ $\eta$, there exists $n_{0} \in \mathbb{N}$ such that $\varphi^{n_{0}}(d(x, y))<\eta<\eta$. Since $\lim _{n \rightarrow \infty} \phi^{n}\left(d\left(x_{0}, x_{1}\right)\right)=\eta$, there exists $m_{0} \in \mathbb{N}$ such that $\eta<\phi^{m}\left(d\left(x_{0}, x_{1}\right)\right)<\delta+\eta$, for all $m>m_{0}$. Thus, we conclude that $\varphi^{m_{0}+n_{0}}\left(d\left(x_{0}, x_{1}\right)\right)<\eta$, a contradiction. So $\lim _{n \rightarrow \infty} \phi^{n}\left(d\left(x_{0}, x_{1}\right)\right)=0$, that is, $\lim _{n \rightarrow \infty} d\left(x_{n}, x_{n+1}\right)=0$.

Next, we claim that $\left\{x_{n}\right\}$ is a Cauchy sequence. We claim that the following result holds:

for each $\varepsilon>0$, there is $n_{0}(\varepsilon) \in \mathbb{N}$ such that for all $m, n \geq n_{0}(\varepsilon)$,

$$
d\left(x_{m}, x_{n}\right)<\varepsilon, \quad(* *)
$$

We shall prove $(* *)$ by contradiction. Suppose that $(* *)$ is false. Then there exists some $\varepsilon>0$ such that for all $p \in \mathbb{N}$, there are $m_{p}, n_{p} \in \mathbb{N}$ with $m_{p}>n_{p} \geq p$ satisfying:

(i) $d\left(x_{m_{p}}, x_{n_{p}}\right) \geq \varepsilon$, and

(ii) $m_{p}$ is the smallest number greater than $n_{p}$ such that the condition $(i)$ holds.

Since

$$
\begin{aligned}
\varepsilon & \leq d\left(x_{m_{p}}, x_{n_{p}}\right) \\
& \leq d\left(x_{m_{p}}, x_{m_{p-1}}\right)+d\left(x_{m_{p-1}}, x_{n_{p}}\right) \\
& \leq d\left(x_{m_{p}}, x_{m_{p-1}}\right)+\varepsilon,
\end{aligned}
$$

hence we conclude $\lim _{p \rightarrow \infty} d\left(x_{m_{p}}, x_{n_{p}}\right)=\varepsilon$. Since

$$
d\left(x_{m_{p}}, x_{n_{p}}\right)-d\left(x_{m_{p}}, x_{m_{p+1}}\right) \leq d\left(x_{m_{p}+1}, x_{n_{p}}\right) \leq d\left(x_{m_{p}}, x_{m_{p}+1}\right)+d\left(x_{m_{p}}, x_{n_{p}}\right),
$$

we also conclude $\lim _{p \rightarrow \infty} d\left(x_{m_{p}+1}, x_{n_{p}}\right)=\varepsilon$. Thus, there exists $i, 0 \leq i \leq k-1$ such that $m_{p}-n_{p}+i=1 \bmod k$ for infinitely many $p$. If $i=0$, then we have that for such $p$,

$$
\begin{aligned}
\varepsilon & \leq d\left(x_{m_{p}}, x_{n_{p}}\right) \\
& \leq d\left(x_{m_{p}}, x_{m_{p}+1}\right)+d\left(x_{m_{p}+1}, x_{n_{p}+1}\right)+d\left(x_{n_{p}+1}, x_{n_{p}}\right) \\
& \leq d\left(x_{m_{p}}, x_{m_{p}+1}\right)+\varphi\left(d\left(x_{m_{p}}, x_{n_{p}}\right)\right)+d\left(x_{n_{p}+1}, x_{n_{p}}\right) .
\end{aligned}
$$

Letting $p \rightarrow \infty$. Then by the condition $\left(\phi_{2}\right)-(a)$ of $\phi$-mapping, we have

$$
\varepsilon \leq 0+\lim _{p \rightarrow \infty} \varphi\left(d\left(x_{m_{p}}, x_{n_{p}}\right)\right)+0<\varepsilon,
$$

a contradiction. The case $i \neq 0$ similar. Thus, $\left\{x_{n}\right\}$ is a Cauchy sequence. Since $X$ is complete, there exists $v \in \cup_{i=1}^{k} A_{i}$ such that $\lim _{n \rightarrow \infty} x_{n}=v$. Now for all $i=0,1,2, \ldots, k$ - $1,\left\{f^{k n-i} x\right\}$ is a sequence in $A_{i}$ and also all converge to $v$. Since $A_{i}$ is closed for all $i=$ $1,2, \ldots, k$, we conclude $v \in \cup_{i=1}^{k} A_{i}$, and also we conclude that $\cap_{i=1}^{k} A_{i} \neq \phi$. By the condition $\left(\phi_{2}\right)-(b)$ of $\phi$-mapping, we have

$$
\begin{aligned}
d(v, f v) & =\lim _{n \rightarrow \infty} d\left(f^{k n} x, f v\right) \\
& \leq \lim _{n \rightarrow \infty} \varphi\left(d\left(f^{k n-1} x, v\right)\right)=0,
\end{aligned}
$$


hence $v$ is a fixed point of $f$. Let $\mu$ be another fixed point of $f$. Since $f$ is a generalized cyclic weaker Meir-Keeler $\phi$-contraction, we have

$$
\begin{aligned}
d(v, \mu) & =d(v, f \mu)=\lim _{n \rightarrow \infty} d\left(f^{k n} x, f \mu\right) \\
& \leq \lim _{n \rightarrow \infty} \varphi\left(d\left(f^{k n-1} x, \mu\right)\right) \\
& <d(\nu, \mu),
\end{aligned}
$$

a contradiction. Therefore, $\mu=v$. Thus $v$ is a unique fixed point of $f$.

Example 8 Let $X=\mathbb{R}^{3}$ and we define $d: X \times X \rightarrow \mathbb{R}^{+}$by

$$
d(x, y)=\left|x_{1}-y_{1}\right|+\left|x_{2}-y_{2}\right|+\left|x_{3}-y_{3}\right|, \text { for } x=\left(x_{1}, x_{2}, x_{3}\right), y=\left(y_{1}, y_{2}, y_{3}\right) \in X,
$$

and let $A=\{(x, 0,0): x \in \mathbb{R}\}, B=\{(0, y, 0): y \in \mathbb{R}\}, C=\{(0,0, z): z \in \mathbb{R}\}$ be three subsets of $X$. Define $f: A \cup B \cup C \rightarrow A \cup B \cup C$ by

$$
\begin{aligned}
& f((x, 0,0))=\left(0, \frac{1}{4} x, 0\right) ; \quad \text { for all } x \in \mathbb{R} ; \\
& f((0, y, 0))=\left(0,0, \frac{1}{4} y\right) ; \quad \text { for all } y \in \mathbb{R} ; \\
& f((0,0, z))=\left(\frac{1}{4} z, 0,0\right) ; \quad \text { for all } z \in \mathbb{R} .
\end{aligned}
$$

and define $\phi: \mathbb{R}^{+} \rightarrow \mathbb{R}^{+}$by

$$
\varphi(t)=\frac{1}{3} t \quad ; \text { for } t \in \mathbb{R}^{+} .
$$

Then $f$ is a generalized cyclic weaker Meir-Keeler $\phi$-contraction and $(0,0,0)$ is the unique fixed point.

\section{Acknowledgements}

The authors would like to thank referee(s) for many useful comments and suggestions for the improvement of the article.

\section{Competing interests}

The author declares that they have no competing interests.

Received: 3 December 2011 Accepted: 16 March 2012 Published: 16 March 2012

\section{References}

1. Kirk, WA, Srinivasan, PS, Veeramani, P: Fixed points for mappings satisfying cyclical contractive conditions. Fixed Point Theory. 4(1):79-89 (2003)

2. Karpagam, S, Agrawal, S: Best proximity point theorems for cyclic orbital Meir-Keeler contraction maps. Nonlinear Anal. 74, 1040-1046 (2010)

3. Edelstein, M: On fixed and periodic points under contractive mappings. J Lon Math Soc. 37, 74-79 (1962). doi:10.1112/ jlms/s1-37.1.74

4. Geraghty, MA: On contractive mappings. Proc Am Math Soc. 40, 604-608 (1973). doi:10.1090/S0002-9939-1973-03341765

5. Boyd, DW, Wong, SW: On nonlinear contractions. Proc Am Math Soc. 20, 458-464 (1969). doi:10.1090/S0002-9939-19690239559-9

6. Meir, A, Keeler, E: A theorem on contraction mappings. J Math Anal Appl. 28, 326-329 (1969). doi:10.1016/0022-247X (69)90031-6

7. Di Bari, C, Suzuki, T, Vetro, C: Best proximity for cyclic Meir-Keeler contractions. Nonlinear Anal. 69, 3790-3794 (2008). doi:10.1016/j.na.2007.10.014

8. Jankovic, S, Kadelburg, Z, Radonevic, S, Rhoades, BE: Best proximity point theorems for p-cyclic Meir-Keeler contractions. Fixed Point Theory Appl 2009, 9 (2009). Article ID 197308 
9. Suzuki, T: Fixed-point theorem for asymptotic contractions of Meir-Keeler type in complete metric spaces. Nonlinear Anal. 64, 971-978 (2006). doi:10.1016/j.na.2005.04.054

10. Suzuki, T: Moudafis viscosity approximations with Meir-Keeler contractions. J Math Anal Appl. 325, 342-352 (2007) doi:10.1016/j.jmaa.2006.01.080

doi:10.1186/1687-1812-2012-41

Cite this article as: Chen: Fixed point theorems for cyclic Meir-Keeler type mappings in complete metric spaces. Fixed Point Theory and Applications 2012 2012:41.

Submit your manuscript to a SpringerOpen ${ }^{\odot}$ journal and benefit from:

- Convenient online submission

- Rigorous peer review

- Immediate publication on acceptance

- Open access: articles freely available online

- High visibility within the field

- Retaining the copyright to your article

Submit your next manuscript at $\boldsymbol{\nabla}$ springeropen.com 